\title{
Die ersten 100 Tage im Beruf - oder wie ich schwimmen lernte
}

\author{
Dr. Esther Hartwich \\ Beisitzerin im Landesverband Berlin im djb; Referatsleiterin Bil- \\ dungsrecht beim DIHK - Deutscher Industrie- und Handelskam- \\ mertag e.V., Berlin
}

Im Sommer 2008 stand ich zum ersten Mal in meinem Leben in meinem eigenen Büro mit meinem Namen an der Tür wow, dachte ich, geschafft! Da ahnte ich noch nicht, dass die eigentliche Herausforderung noch vor mir lag ...

Durch das Referendariat war mir klar geworden, dass ich lieber in einem politischen als in einem rein juristischen Bereich arbeiten wollte, weshalb Verbände für mich eine attraktive Arbeitsmöglichkeit darstellten. Unter den rund 40 Bewerbungen, die ich innerhalb von drei Monaten rausschickte, war auch eine an den DIHK (Deutscher Industrieund Handelskammertag) gerichtet, der eine „Referentin für das Bildungsrecht" suchte. Ich hatte zwar kaum Ahnung vom Bildungsrecht oder davon, was der DIHK oder eine IHK so genau macht, aber die Stelle klang interessant, vielseitig und nicht ausschließlich juristisch. Und siehe da, nach fünf Gesprächsrunden, die sich über einen Monat hinzogen, hatte der DIHK sich tatsächlich für mich und ich mich für den DIHK entschieden.

Und da war ich nun, „Referatsleiterin für Bildungsrecht“, und war gespannt, was alles auf mich zukommen würde. Und das war eine ganze Menge! Nach dem ersten „Wow! Ich habe es geschafft-Gefühl“ kam ziemlich schnell der Eindruck, ins kalte Wasser geschubst zu werden und nicht so richtig zu wissen, wo denn nun die Oberfläche ist. Das fing damit an, sich in ein fremdes Rechtsgebiet einzuarbeiten, ging darüber, sich in die Struktur der IHK-Organisation einzufinden, und endete bei der Feststellung, dass man auf keinen Fall eine fremde Teetasse benutzen darf.

Die Arbeit in einem mir nicht vertrauten Rechtsgebiet war dabei das geringste Problem - schließlich hat man als Juristin ja genau diese Fähigkeit erlernt. Mein neues Arbeitswerkzeug wurde das Berufsbildungsgesetz (BBiG), das die berufliche Aus- und Weiterbildung in Deutschland regelt. Was ich vorher nicht wusste: Der betriebliche Teil der dualen Ausbildung in Deutschland wird von der örtlich zuständigen IHK begleitet und überwacht und die Zwischen- und Abschlussprüfungen werden in der beruflichen Aus- und Weiterbildung abgenommen. Bei rund 900.000 Auszubildenden und 120.000 Teilnehmern von Weiterbildungsprüfungen pro Jahr ist das ein riesiges Tagesgeschäft, das auch eine Menge rechtlicher Fragen mit sich bringt. Beim DIHK gibt es deshalb zwei Jurist(inn)en, die den IHKs beratend zur Seite stehen. Eine davon war jetzt ich und so wurde ich ab dem ersten Tag mit den verschiedensten Fragen rund um dieses Themengebiet konfrontiert. Dass die IHK-Kolleg(inn)en die Antwor- ten nicht nur sofort, sondern insbesondere für Nichtjurist(inn) en verständlich und mit einer praxisnahen Umsetzungsempfehlung haben wollten, merkte ich schnell an den irritierten Reaktionen meiner „Kunden“, wenn ich zu sehr ins formaljuristische abwich.

Das Einfinden in die IHK-Organisation war da schon komplizierter. 80 IHKs gibt es bundesweit, die rund 3,6 Millionen Unternehmen in Deutschland vertreten. Vor hundert Jahren wurde der heutige DIHK gegründet, der als Dachverband mit rund 180 Mitarbeiter(inne)n in Berlin und Brüssel die bundesweite Koordination der IHKs übernimmt sowie für die bundes- und europapolitische Vertretung zuständig ist. Dazu kommt die Arbeit der AHKs an 120 Standorten in 80 Ländern weltweit. Da soll man sich erstmal zurechtfinden ... Auch wenn das „Information-Overload-Gefühl“ sich in der ersten Zeit einfach nicht vermeiden lässt, war es sehr hilfreich, dass der DIHK für alle neue Mitarbeiter(innen) nicht nur Gespräche mit Kolleg(inn)en aus dem eigenen Bereich, sondern auch mit Vertreter(inne)n aus allen anderen Bereichen sowie Stagen in Brüssel und bei einer IHK vorsah. Insbesondere die Stage bei der IHK fand ich sehr wichtig und hilfreich, denn schließlich ist es eines der Hauptaufgaben eines Dachverbandes, zu koordinieren und seine Mitglieder zu unterstützen. Aber wie bringt man die Interessen von 80 IHKs und damit letztlich 3,6 Millionen Unternehmen unter einen Hut? Und das auch noch ohne jede Weisungsbefugnis oder ein Direktionsrecht? Nach 100 Tagen war meine Antwort ganz klar: nur mit viel Geduld, Sensibilität und einem sehr, sehr dicken Fell.

Ebenso klar war nach den ersten 100 Tagen, dass ich mich in diesem Job zumindest nie über Langeweile oder fehlende Vielseitigkeit beklagen können würde. Denn neben der juristischen Beratung und der klassischen Verbandsarbeit gehörte es auch zu meinen neuen Aufgaben, Artikel zu schreiben, Vorträge zu halten, Seminare zu geben, der Presse für Interviews zur Verfügung zu stehen und ein öffentlich gefördertes Projekt mit zu begleiten. Ich bin nicht mehr sicher, ob ich schon nach 100 Tagen das Gefühl hatte, aus dem Wasser aufgetaucht und die Oberfläche wieder erreicht zu haben. Bis ich das Gefühl hatte, sicher schwimmen zu können, hat es aber auf jeden Fall länger gedauert. Was mir dabei geholfen hat? Die Unterstützung der Kolleg(inn)en und meiner Chefin sowie der Mut, „einfach mal zu machen“, und die Bereitschaft, aus Fehlern, die sich am Anfang einfach nicht vermeiden lassen, zu lernen. Übrigens sind das Dinge, die nach nun mittlerweile 900 Tagen im Beruf noch genauso gelten wie in den ersten 100 Tagen. 